\title{
HST AND KECK OBSERVATIONS OF HIGH REDSHIFT RADIO GALAXIES
}

\author{
W.J.M. VAN BREUGEL \\ Institute of Geophysics and Planetary Physics \\ Lawrence Livermore National Laboratory \\ P.O. Box 808, L-413, Livermore, CA 94550, USA
}

\section{Introduction}

Together with several of my colleagues I have embarked on a comprehensive program to study the radio-aligned restframe UV structures in high redshift radio galaxies ( $\mathrm{HzRGs}$ ) using some of the world's premier optical telescopes: the Hubble Space Telescope for high spatial resolution imaging, and the Keck $10 \mathrm{~m}$ telescope for high $\mathrm{S} / \mathrm{N}$ spectropolarimetry. I will discuss some of our latest results from these observations which elucidate, and at the same time obscure, our evolving understanding of HzRGs .

\section{HST Imaging of $4 \mathrm{C} 41.17$ at $\mathrm{z}=\mathbf{3 . 8 0 0}$}

4C41.17 is the highest redshift radio galaxy observed with the refurbished HST to date. Using the WFPC2 with the F702W and F569W filters respectively a deep, line free restframe UV (1500 $\AA)$ image and a Ly- $\alpha$ emission line image were obtained.

\subsection{ALIGNED UV CONTINUUM AND Ly- $\alpha$ EMISSION}

The rest-frame UV continuum of $4 \mathrm{C} 41.17$ has a complex, elongated morphology with numerous compact (kpc- sized), bright $(\sim 0.2 \mu \mathrm{Jy}$; ' $R$ ' $=$ 26.7) components which are aligned, but not coincident, with the inner radio source. The radio core (AGN) is located in a gap between two main regions, both of which are embedded in faint, diffuse emission. This suggests that the AGN is probably obscured, at least in the rest-frame UV. 
Imaging and spectropolarimetry of HzRGs, including recent high signalto-noise spectropolarimetry with Keck (these Proceedings), show strong evidence that a significant fraction of the aligned rest-frame UV continuum in these radio galaxies is scattered light from obscured or 'misdirected' quasarlike AGN. Thus, by analogy, it seems that at least some of the aligned UV continuum in $4 \mathrm{C} 41.17$ has a similar origin. The total luminosity of the aligned clumps in $4 \mathrm{C} 41.17$ is $\log \left[\nu L_{\nu, \text { aligned }}\right]=46.9\left(\mathrm{erg} \mathrm{s}^{-1}\right)$ which is comparable to that of radio loud $1<z<2$ quasars at this rest wavelength $\left(46.4<\log \left[\nu L_{\nu}\right]=47.6\right)$. Thus on purely energetic considerations the aligned rest-frame UV in $4 \mathrm{C} 41.17$ may indeed be due to collimated, scattered light from a hidden quasar-like AGN.

The Ly- $\alpha$ emission in 4C41.17 does not appear to be directly associated with the UV continuum knots, with a possible exception in the western region of $4 \mathrm{C} 41.17$. Instead the line emission seems brightest near the boundaries of the radio components, a feature which is often observed in nearby radio galaxies and which suggests interaction (entrainment, ionization) of the radio source with relatively dense ambient gas. Support for such an interpretation follows also from the high-velocity component seen in $4 \mathrm{C} 41.17$, with a total range of $2000 \mathrm{~km} \mathrm{~s}^{-1}$ over the total extent (several arcseconds) of the inner radio source. The dominant source of ionization, AGN, shocks, or starformation is unclear at present, but the arguments above for the probable presence of a hidden quasar in $4 \mathrm{C} 41.17$ suggest that photoionization by the AGN is likely. The total Ly- $\alpha$ flux of the aligned component in the HST image is $F_{L y a}=1.2 \times 10^{-15} \mathrm{erg} \mathrm{s}^{-1} \mathrm{~cm}^{-2}$, implying a Ly- $\alpha$ luminosity of $\log \left[L_{L y a}\right]=44.2 \mathrm{erg} \mathrm{s}^{-1}$, which is comparable to that of steep spectrum quasars.

\subsection{COMPANION GROUP}

There is an amorphous, 'non-aligned', clumpy group of objects south of 4C41.17 which is embedded in a halo of diffuse continuum emission and which appears connected with the $4 \mathrm{C} 41.17$ system. Keck near-IR images have shown that this companion system is very blue $\left(\alpha_{1500 \AA} \sim 0\right)$ and has a luminosity comparable to that of a $M_{B}=-20.5 L_{*}$ galaxy at this redshift. Thus the companion group may be a galaxy sized system with ongoing star formation. The individual clumps in this system have UV luminosities which are $10-100$ times higher than those of blue compact dwarf galaxies, in a similar kpc-sized volume, indicating vigorous star formation indeed. The HST observations of $4 \mathrm{C} 41.17$ therefore suggest that we may be viewing, for the very first time, the formation of a galaxy through merging of kiloparsec sized, very active starforming regions ('building blocks'), as predicted in dissipative galaxy formation scenarios. These star formation 


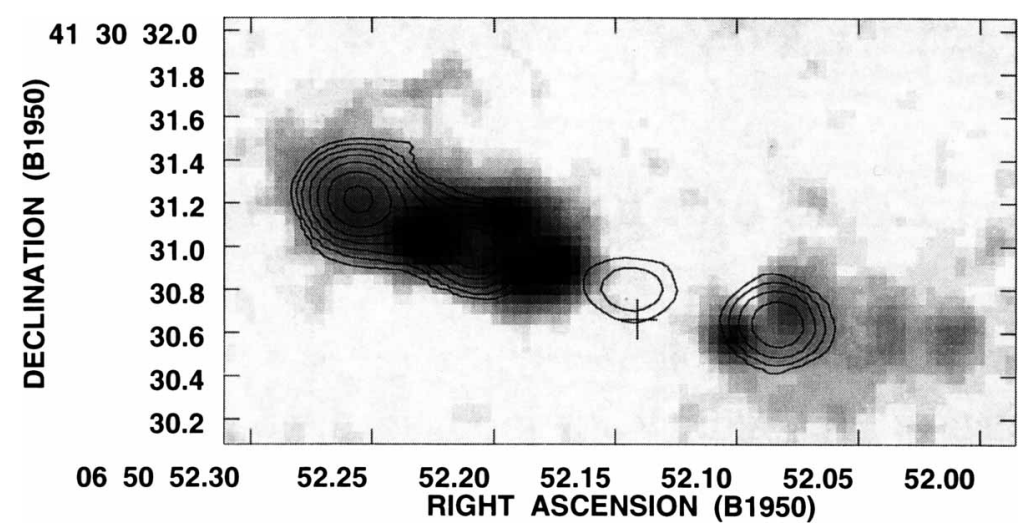

Figure 1. HST WFPC2 image of 4C41.17 with radio contours superimposed

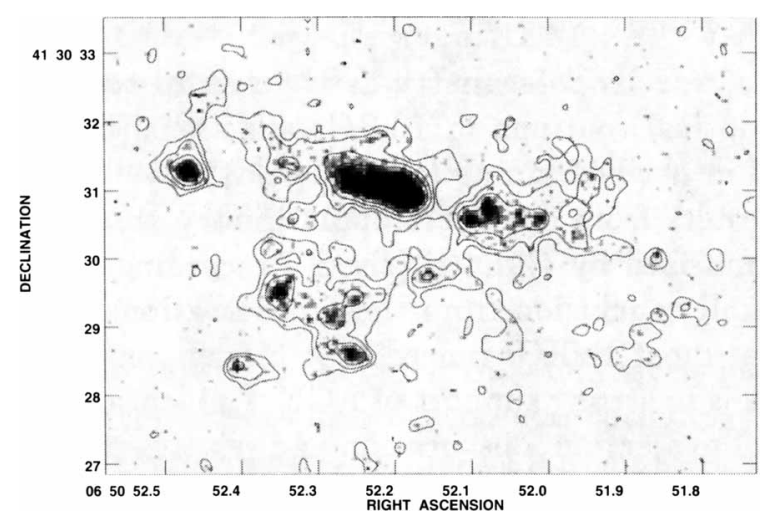

Figure 2. Same data as Fig.1 showing the clumpy companion system

clumps could be sources for dust and dense gas and thus, for example, provide scattering mirrors or ionization material as they are intercepted by the collimated UV-continuum radiation from the 4C41.17 AGN. The AGN itself, one might hypothesize, could have formed through rapid stellar evolution and the formation of a black hole in the center one of these clumps, possibly triggered by the merging process.

\section{Keck Spectropolarimetry of HZRGs}

During the past few years there has been increasing evidence that aligned restframe UV $(<4000 \AA)$ continua in HzRGs may be dominated by scattered light from hidden or mis-directed AGN. The large aperture of the Keck telescope, combined with the often excellent seeing, allows one to obtain vastly superior, high $\mathrm{S} / \mathrm{N}$ and spatially resolved spectropolarimetry data of HzRGs . Now, for the first time, it has become possible to use the 


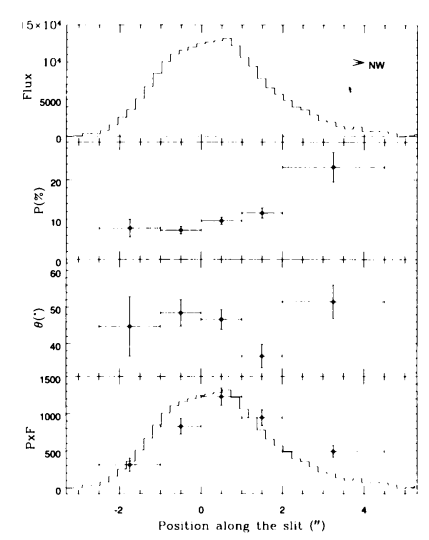

Figure 3. Total flux, percentage polarization, position angle, and polarized flux along the radio axis of $3 \mathrm{C} 256$ observed at Keck

full potential of spectropolarimetry as a powerful tool in discovering the nature of the optical continua in HzRGs and their (hidden) AGN, and to probe the ISM in newly forming (active) galaxies.

The first results from our spectropolarimetry observations program at Keck are summarized by Cimatti (these Proceedings). Perhaps the most important overall conclusion from these observations is that there is now little doubt that most HzRGs indeed have hidden, or 'mis-aligned', quasarlike AGN. This is in strong support of AGN 'Unification' models which rely on the effects of projection, obscuration and relativistic beaming to explain many of the observed properties of various classes of active galaxies. Here I would like to emphasize two additional points:

- The spatial extents of the radio/UV-aligned reflection nebulae of HzRGs can be enormous ( $5^{\prime \prime}$, or $55 \mathrm{kpc}$ in $3 \mathrm{C} 256$ at $z=1.825$ [Dey et al. 1995]).

- While a large fraction of the UV continuum is shown to be polarized in HzRGs, a significant fraction is un-polarized. Furthermore probably not all HzRGs are highly polarized (preliminary analysis of our HST imaging polarimetry and Keck spectropolarimetry shows that the percentage polarization in the proto-type aligned radio galaxy 3C368 may in fact be much less than previously thought). Thus now the interesting question arises as to what the origin is of this unpolarized continuum. Nebular continuum associated with strong emission-line regions appears as an attractive possible source (Tadhunter, these Proceedings).

The work by $W v B$ described here was performed at IGPP/LLNL under the auspices of the U.S. Dept. of Energy under contract W-7405-ENG-48. It is in collaboration with Miley, McCarthy, and Spinrad (4C41.17), and with Dey, Cimatti, Antonucci, and Spinrad (Keck spectropolarimetry), who I thank for allowing me to use these data prior to publication. 